\title{
Inverted L-Shaped Nasal Strut as a Modified Septal Extension Graft
}

\author{
AYMAN M. ABDELHAMID, M.D.; SHERIF MAHER, M.D. and WALEED ALDABAANY, M.D. \\ The Department of Plastic Surgery, Faculty of Medicine, Fayoum University, Fayoum, Egypt
}

\begin{abstract}
Background: Correction of the nasal tip in rhinoplasty surgery with better tip support needs a considerable effort. For that issue, we used certain technique which is the use of L-shaped columellar strut fixed in inverted position as septal extension graft for better tip projection and fixation.
\end{abstract}

Aim of Work: Assessment of the use of inverted L-shaped columellar strut in rhinoplasty surgery for correction of nasal tip shape and position with more stable nose.

Patients and Methods: This study included 15 patients who are candidate for rhinoplasty surgery for correction of nasal tip. They underwent surgery through open approach rhinoplasty in which special collumellar strut used as septal extension graft to achieve good tip projection with modification of the site of fixation to the caudal septum according to additional nasal deformity present. Assessment of patients done according to the photographs taken and patient's satisfaction.

Results: Of the 15 patients who have undergone this procedure, 15 were available for follow-up. 7 patients showed excellent results, 6 patients were considered as a good outcome and 2 patients were considered as fair results.

Conclusion: Inverted L-shaped collumellar struts used as septal extension graft proved to be effective to correct nasal tip shape, position and stability.

Key Words: Inverted L shaped Strut - Septal extension graft.

\section{INTRODUCTION}

Nasal tip reinforcement is an important consideration for rhinoplasty operationshence it is required in most cases. The nasal tip supported by a number of soft tissue components and cartilaginous relationships [1]. The attachments that connect the lateral crura and the medial crura with the nasal septum, and that of Pitanguy's dermocartilaginous ligament all play important rolesfor the many techniques for improving tip projection [2].

The degree to which the height and length of the nasal tip can be increased and maintained to enhance the aesthetic quality of the nose is surely one of the major challenges and concerns for plastic surgeons. Furthermore, since the naturalness of the nasal tip must not be damaged, surgeons apply various methods using autologous materials to solve this problem [3].

Although septal extension graft is one of the most popular techniqueshowever, it has some disadvantages as thenasal tip projection and rotation are less. However, this graft often results in stiffness of the nasal tip, not all patients have enough septal cartilage for proper extension, and the amount of septal cartilage harvested may be relatively small in many cases. In addition, in secondary cases, the septal cartilage may have been used before [4].

Caudal septal extension grafts are segments of perpendicular cartilage which are secured to the caudal septum and extend into the columella. The caudal segment of septal cartilage provides architectural support to the nasal tip. Loss of that caudal septal support will substantially affect the stability of the nasal tip and thereby result in aesthetic as well as functional problems [5].

Thus, the main purpose of the caudal septal extension grafts is to stabilize the nasal base and tip. The dimension of the graft may vary from a medium-sized strut to a large plate of several centimetres side length [6]. The caudal septal extension graft creates a firm conjunction between the septum and the columella-tip complex. By suturing the medial crura to the graft in the desired position, the surgeon is enabled to definitely determine tip and columella position in terms of projection, rotation and nasolabial angle [7].

The caudal septal extension graft is the most powerful tool to hold the tip in a definitive position. Therefore, it is especially useful when the corrected nose must with stand strong tractional and gravitational forces in the long term, as it is the case in correction of an under projected and under rotated nasal tips with heavy soft tissue mantle. The graft proved to be effective in restoring the lost tip 
support in patients with caudal septal deficiency [8].

For an optimal outcome, we propose a design of an L-shaped columellar strut, which requires a smaller amount of cartilage and serves as a powerful tool for controlling tip projection and rotation, reached the same target by replacing the a large plate of several centimeters side length graft by using one piece of cartilage L-shape fixed upside down as strut and works additionally as septal extension graft.

\section{PATIENTS AND METHODS}

This study included 15 patients who are candidate for correction of nasal tip deformity. Who attended the Outpatient Clinic Plastic Surgery Department Fayoum University Hospitals in the period between March 2016 to March 2017. All of the patients who underwent the L-shaped columellar strut graft using septal cartilage in this study were primary rhinoplasty cases.Preoperative examination was con-ducted to obtain a detailed history regarding presenting complaints, history of present illness with special note of presence or absence of symptoms like nasal obstruction, nasal discharge, hyposmia, sneezing, nasal bleeding and postnasal drip was taken. The detailed general physical examination and examination of nose and throat were carried out. The evaluation of the operation results was made by comparing the preoperative and postoperative photos of the nasal tip position, angles and projection.

\section{Surgical technique:}

The operations took place under general anesthesia with orotracheal intubation and local-regional infiltration with 2\% Xylocaine and 1:100,000 adrenaline. Surgery was begun after about 15 min. Standard rhinoplastic methods were used like, septoplasty, tip correction, hump removal, augmentation according to the situation of patient.

Open rhinoplasty was performed in all of the cases with a ' $V$ ' shape incision in the columella with a bilateral marginal incision. Both the cartilaginous and the bony sections of the nasal dorsum are carefully detached down to the subperichondrial and subperiosteal level. The two medial crura are drawn out and an incision is then made in the intercrural tissue to reach the most proximal section of the caudal septum. The next step is the subperichondrial detachment of both sides of the nasal septum. In exposing the basal region of the septum, it also proves useful to expose the region of the nasal spine.
Cartilage was taken from the septum to design special collumellar strut (L-shape) (Fig. 1) used as septal extension graft to achieve good tip projection. The width (cephalocaudal dimension) of the transverse part of L-strut will be longer than the width of the medial crura plus the deficiency in nasal length by millimetres to allow for fixation to the caudal septum with the vertical part dimensions equal to the usual strut. Modification of the angle of the L-strut to be more acute to allow fixation of the short limb of L-strut to the vertical caudal part or the anterocaudal angle of the septum to avoid widening of the dorsum (Fig. 2). This modification of the site of fixation to the caudal septum is done according to additional nasal deformity present. These grafts are sewn in position to the septum using 5-0 prolene in at least two sites. The horizontal portion of the strut was fixed to the caudal septum with more than two sutures and thevertical portion of the strut was then placed between the medial crurae of the lower lateral cartilages.

Hump excision was performed at this point, ifrequired, followed by medial and lateral microosteotomies, two of which are often performed on each side toeliminate any asymmetry of the bony structure (Fig. 3).

Following the surgery we inserted nasal pack for 48 hours and then removed, and steri-strips \& nasal splint was left for 8 days.
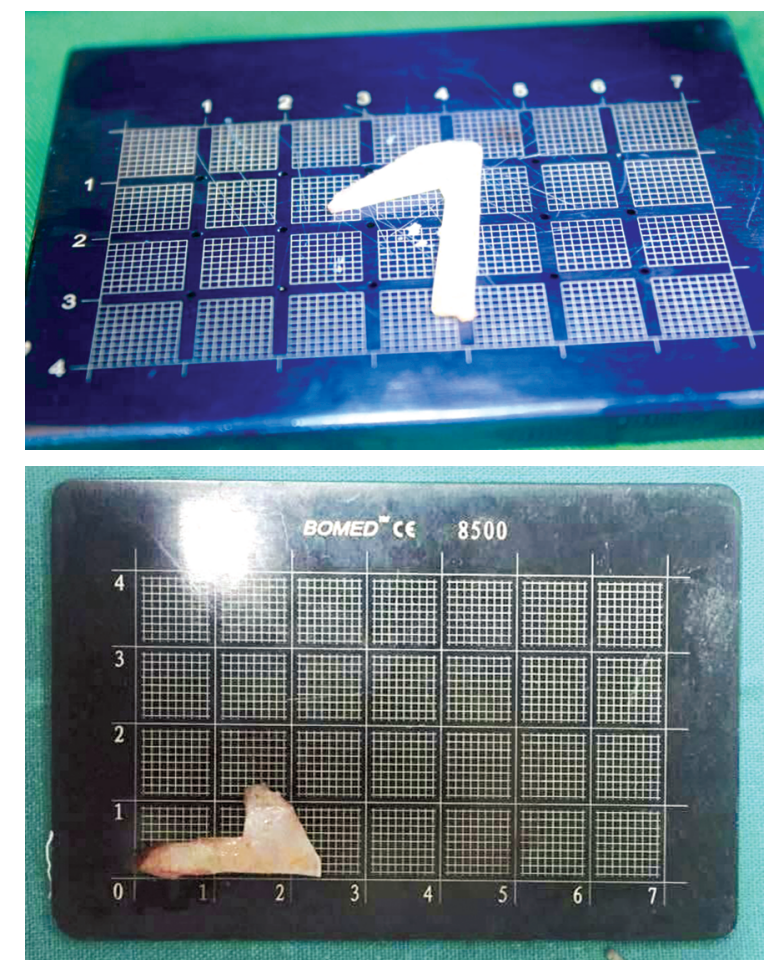

Fig. (1): The cartilage was carvedinto an L-shaped strut. 




Fig. (2): Septal cartilage was fixed with an L-shaped strut.

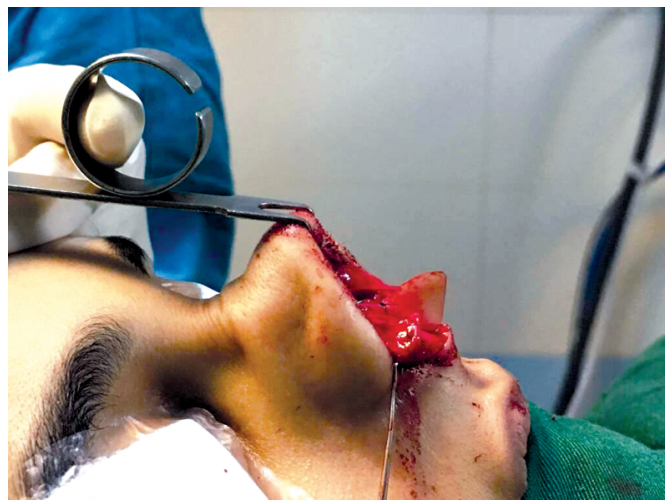

Fig. (3): The inverted L-shaped strut in place after fixation.

\section{RESULTS}

Out of the 15 patients included in this study, 12 were women $(80 \%)$ and 3 were men $(20 \%)$; their mean age was 33.5 years (range, 20-45) years. All cases were primary rhinoplasty. The duration of follow-up was at least six months. Aesthetic results were satisfactory to both patients and surgeon. In this study tip correction was done in all cases. More procedures were done in same patient in 11 cases include dorsal hump excision and correction of abnormal naso-labialangle. On evaluating the degree of patient satisfaction, 13 patients $(86.6 \%)$ were satisfied with their aesthetic and functional improvement; no case requested further revision for correction of minor deformities (Fig. 4).

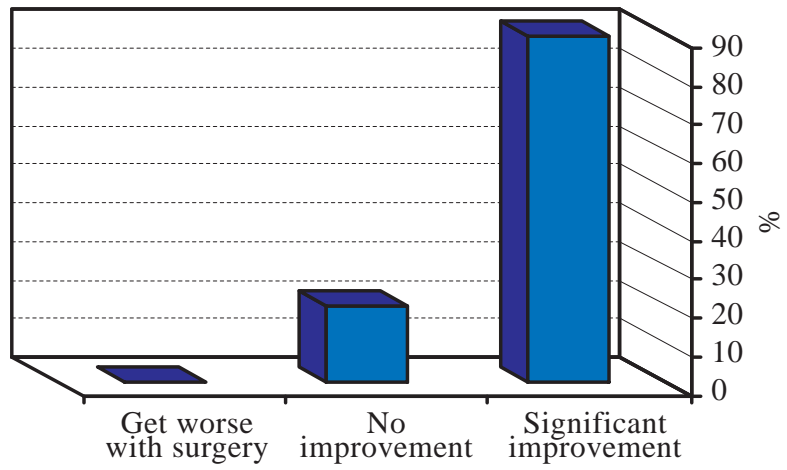

Fig. (4): The degree of improvement for patients after Lshaped Nasal strut.
On evaluating the post operative period and complications the oedema and ecchymosis subsided markedly after removal of the splint. In 3 patients the oedema lasted for two months following surgery. Short term post operative complication like septal hematoma, haemorrhage and infection was not detected in any patient.

Seven patients $(46.67 \%)$ showed excellent results (more than $80 \%$ improvement), six patients $(40 \%)$ were considered as a good outcome (40$80 \%$ improvement), and two patients $(13.3 \%)$ attained fair results (less than $40 \%$ improvement) after asking the patients about the percentage of improvements and satisfaction (Figs. 5,6).
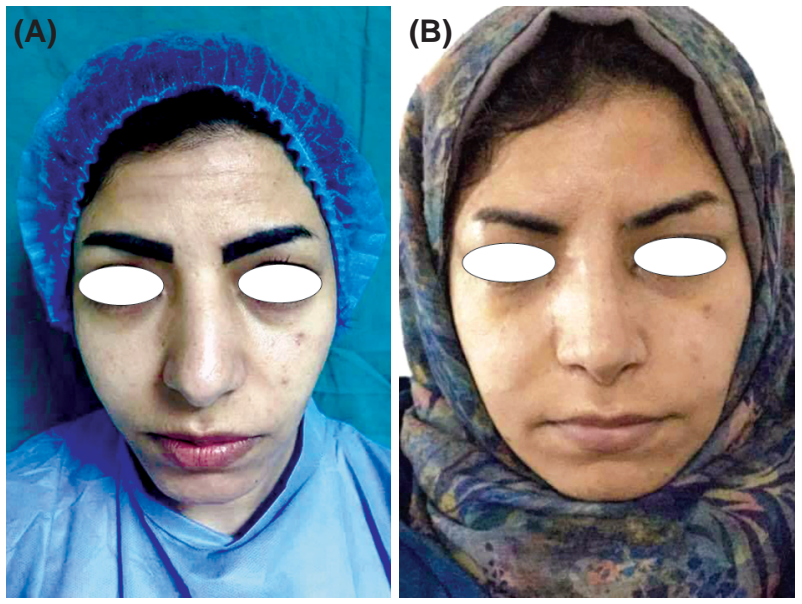

Fig. (5): Frontal view of patient with L strut. (A): Preoperative (B): Postoperative.
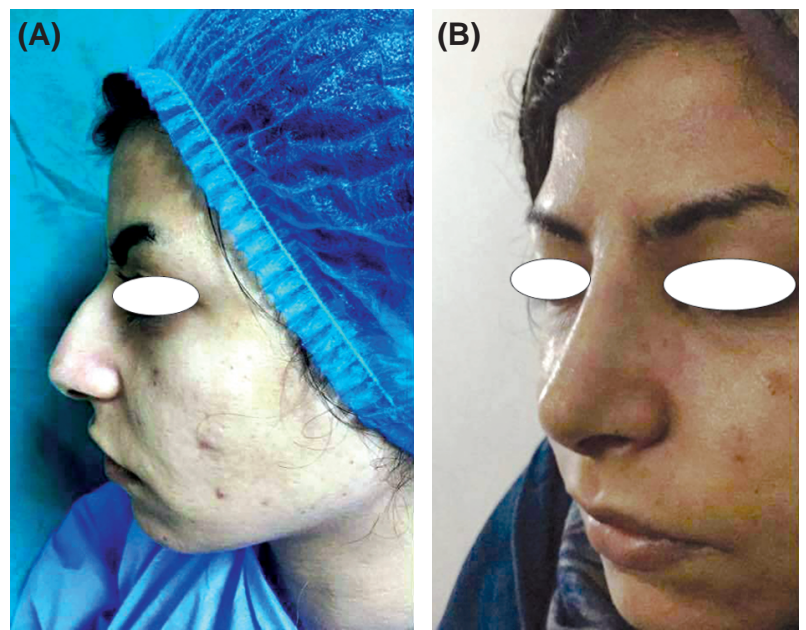

Fig. (6): Side view of patient with L strut. (A): Preoperative (B): Postoperative.

\section{DISCUSSION}

Correction of nasal tip shape, position and support has a cardinal role in the long term result of rhinoplasty surgery, multiple techniques have been used to get better nasal tip support, augmen- 
tation and maintain nasolabial angle. Septal extension graft is one of those commonly used methods for defining the nasal tip. Analysis of preoperative and postoperative results of columellar struts showed that tip projection was sufficiently controlled only with fixation of the strut to the caudal septum and to the medial crurae [9].

Nasal septal cartilage is the primary source for cartilage grafts and if not possible as in cases of previous septoplasty, anatomically insufficient cartilage or septal cartilage ossification, then autologous rib cartilage can be used, Some authors as Han et al. [10] used foreign material; Porous high density polyethylene sheets in Asian rhinoplasty as septal extension graft, others used nasal bony septum with special concern of faster absorption, difficult fixation and easily fractured than septal cartilage graft [11].

When using septal extension graft alone or nasal strut alone for lengthening and projection of the nose, the summation force (gravity, scar contraction and the generated reaction force) leads to decrease of projection, shift the tip to either side of the caudal septum resulting in collapse and deviation of the nasal tip or failure of nasal elongation.

So, additional support to the strut or modification of the septal extension graft is required to maintain tip projection, with special concern in cases with caudal septum deviation as septal extension grafts may exaggerate these deviations, in these cases use of bilateral spreader grafts provide additional cartilage reinforcement with good tip projection than columellar strut alone but resulting in widening of the nasal dorsum.

Techniques that advocate the use of septal extension graft alone or spreader grafts alone harbour the same disadvantage. Our technique is versatile and avoids the adversities posed by other methods by Modification of the angle of the Lstrut to be more acute to allow for fixation of the short limb of L-strut to the vertical caudal part or the anterocaudal angle of the septum to avoid widening of the dorsum unless used as extension to spreader graft to correct nasal deviation where in such cases the angle made less acute or right angle with long transverse limb of the strut to be used as spreader graft with additional spreader graft on the other side to correct deviation of the dorsum if needed.This modification of the site of fixation to the caudal septum is done according to additional nasal deformity present.

So in cases of nasal dorsum deviation with an unavoidable need to use spreader grafts to correct deviation and strut to add more tip support and projection; this can be done with our technique with less suture fixation.

But if no deviation in the nose which is the role in our study; the angle of the strut is made more acute to allow for fixation, projection and support without widening of the dorsum with better stability and less rigidity (as right angle of L strut or strut fixed between two spreader grafts is more rigid and less resistant to fracture than acute angle one).

The degree of nasolabial angle and nasal tip rotation during surgery can be easily attempted with our technique be changing point of fixation of the L-strut to the septum with more stability.

\section{Conclusion:}

We found that this technique architecturally and aesthetically sound and we recommend it for consideration when nasal tip support, rotation and lengthening is part of the aesthetic objectives.

\section{REFERENCES}

1- Myung-Hoon Kim, Jeong-Hwan Choi, Min-Su Kim, SeokKwun Kim and Keun-Cheol Lee: An Introduction to the Septal Extension Graft Arch. Plast .Surg., 41: 29-34, 2014.

2- Ha R.Y. and Byrd H.S.: Septal extension grafts revisited: 6-year experience in controlling nasal tip projection and shape. Plast. Reconstr. Surg., 112: 1929-35, 2003.

3- Jae Hoon Kim, Jin Woo Song, Sung Wan Park, Won Suk Oh and Joo Heon Lee: Effective Septal Extension Graft for Asian Arch. Plast. Surg., 41: 3-11, 2014.

4- Toriumi D.M.: New Concepts in Nasal Tip Contouring. Archives of Facial Plastic Surgery, 8 (3): 156-85, 2006.

5- Foda H.M.: The Caudal Septum Replacement Graft. Archives of Facial Plastic Surgery, 10 (3): 152-7, 2008.

6- Petroff M.A., Mccollough E.G., Horn D. and Anderson JR.: Nasal Tip Projection: Quantitative Changes Following Rhinoplasty. Archives of Otolaryngology Head and Neck Surgery, 117 (7): 783-8, 1991.

7- Hwang S.H. and Hwang K.: Supporting strength of septal extension grafts. J. Craniofac. Surg., 22: 23-6, 2011.

8- Kim J.S., Choi J.H., Choi T.H., et al.: Modified septal extension graft for the correction of nasal tip and columella. J. Korean Soc. Plast. Reconstr. Surg., 33: 681-7, 2006.

9- Byrd H.S., Andochick S., Copit S. and Walton K.G.: Septal Extension Grafts: Amethod of Controlling Tip Projection Shape. Plastic and Reconstructive Surgery, 100 (4): 999-1010, 1997.

10- Han K., Jeong J.W. and Kim J.H.: Complete Septal Extension Grafts Using Porous High-Density Polyethylene Sheets for the Esternization of the Asian nose. Plastic and Reconstructive Surgery, 130: 106e-115e, 2012.

11- Peer L.A.: Fate of Autogenous Human Bone Grafts. British Journal of Plastic Surgery, 3 (2): 233-43, 1951. 